Revue d'histoire de l'Amérique française

\#H REVUE D.HISTOIRE DE L'AMÉRIQUE FRANÇAISE

\title{
Les Canadiens veulent conserver le régime seigneurial (2ème partie)
}

\section{Marcel-Joseph}

Volume 7, numéro 2, septembre 1953

URI : https://id.erudit.org/iderudit/301593ar

DOI : https://doi.org/10.7202/301593ar

Aller au sommaire du numéro

\section{Éditeur(s)}

Institut d'histoire de l'Amérique française

\section{ISSN}

0035-2357 (imprimé)

1492-1383 (numérique)

Découvrir la revue

\section{Citer cet article}

Marcel-Joseph (1953). Les Canadiens veulent conserver le régime seigneurial (2ème partie). Revue d'histoire de l'Amérique française, 7(2), 224-240.

https://doi.org/10.7202/301593ar d'utilisation que vous pouvez consulter en ligne.

https://apropos.erudit.org/fr/usagers/politique-dutilisation/ 


\title{
LES CANADIENS VEULENT CONSERVER LE RÉGIME SEIGNEURIAL*
}

\author{
(suite)
}

CHAPITRE II

\section{DE THOMAS DUNN A DALHOUSIE}

\section{SUGGESTION DE SEWELL}

Craig pense seulement à créer une aristocratie - Sewell lui recommande une mutation de tenure - La guerre détourne les esprits.

2. CALDWELL VEUT COMMUER LA TENURE DE SES TERRES

Fin des guerres de l'Empire et émigration - Permission d'opérer la mutation accordée - Objections du conseil exécutif - Permission retirée.

3. SURPEUPLEMENT DES ENVIRONS DE QUÉBEC

Fondation de Val-Cartier - Le problème des chemins Plan pour fonder un nouvel établissement.

\section{Suggestion de Sewell}

Milnes parti, les rênes de l'administration passaient entre les mains du plus ancien membre du conseil exécutif, l'honorable Thomas Dunn; lequel "était un homme modéré et conciliant, sympathique aux Canadiens" et qui "s'appliqua à maintenir l'ordre et la tranquillité dans la province"1. Ce n'est donc pas lui qui aurait entrepris la démolition du système seigneurial.

* Voir, pour première partie de cet article, vol. VII, no 1: 45-63.

1. I. Caron, La Colonisation de la province de Quebec. Les Cantons de l'Est 1791$1815,193$. 
La fondation du Canadien peu de temps après l'arrivée de Craig, l'emprisonnement de Bédard, les dissolutions d'Assemblées, le remplacement de Craig par Prevost, la guerre de 1812, voilà, au dire de Sewell, les principaux événements qui firent ensuite oublier la question des tenures ${ }^{2}$.

Sous Craig, quelque seigneur réussit à faire voter par l'Assemblée, une loi pour indiquer en qui résidait le pouvoir d'accorder des Lettres de Terrier ${ }^{3}$, c'est-à-dire la permission de faire le registre terrier d'une seigneurie, avec tout ce que cela comportait. "Pour empêcher qu'on ne pût invoquer la prescription contre ses droits, le seigneur obtenait du gouvernement de faire exécuter tous les trente ans et même plus souvent, le terrier de sa seigneurie. Les lettres patentes qui l'y autorisaient, étaient lues à trois reprises différentes au prône ou à la porte des églises. Elles obligeaient tous l'es habitants de la seigneurie à venir déclarer d'une manière exacte, au notaire désigné par le seigneur, la nature de leurs terres et la quotité de leurs redevances. Les frais de la rédaction du terrier étaient à la charge des censitaires"4. Depuis 1760 que la question était controversée: les avocats anglais disaient que le droit d'émettre ces lettres, appartenait à la Cour du Banc du Roi et les avocats canadiens à Sa Majesté ou au gouverneur. "A cause de cette incertitude, expliquait Craig, les Seigneurs ont été jusqu'ici dans l'impuissance de forcer leurs censitaires à exhiber leurs Titres, et par suite, ont été frustrés de leurs justes réclamations et mis dans l'impossibilité d'exiger le paiement de rentes qui leur étaient dues en justice. C'est une des causes de l'espèce de dégradation de l'Aristocratie". Voilà encore le refrain. Pour les Anglais de cette époque, un pays ne pouvait prospérer sans aristocratie. "Il est regrettable, ajoutait Craig, que l'influence soit maintenant dans les mains des avocats et des notaires"5. Sewell partageait cette idée; il souffrait de voir les avocats prendre de l'ascendant sur l'Assemblée, au détriment des seigneurs ${ }^{6}$. Craig dési-

2. J. Sewell, "Court résumé de la situation politique du Bas-Canada pendant les derniers sept ans", 12 mai 1814, APC, Q 130-3: 600 .

3. Craig a Castlereagh, 15 juillet 1808, APC, Q 107: 195.

4. J.-E. Roy, Histoire de la seigneurie de Lauzon, 4: 266.

5. Craig à Castlereagh, 15 juillet 1808, APC, Q 107: 196.

6. J. Sewell, "Court résumé de la situation politique du Bas-Canada pendant es derniers sept ans", 12 mai 1814, APC, Q 130-3:618. 
rait corriger cette anomalie. L'émission de lettres de terrier serait de nature à augmenter la valeur de la propriété seigneuriale et à enrichir la petite aristocratie du Canada. Ce serait même un moyen d'augmenter le revenu casuel de la Province, car on pourrait refuser ou différer d'accorder des lettres de terrier aux seigneurs qui n'auraient pas été fidèles à payer leur droit de quint ${ }^{7}$. Ainsi tout serait profit dans cette affaire.

Mais Craig avait assez de soucis par ailleurs, en particulier avec l'achèvement de sa route, sans se préoccuper aussi du changement de tenure. Un autre y pensait cependant, j'ai nommé le vigilant Sewell. Il était toujours là et remplissait même la charge de juge en chef. Craig l'ayant consulté au sujet du malaise qui agitait la Province, il suggéra le remède suivant: une immigration anglaise qu'on disperserait "dans les parties colonisées du pays". Mais cela ne pouvait se faire sans le concours du Parlement. "Toutes les concessions du gouvernement, disait-il, ont été faites en vertu du système féodal... Ce mode de tenure inspire une aversion profonde à tous les Anglais et à tous les Américains, et il s'ensuit que toutes les seigneuries dans la province sont entièrement colonisées par des Canadiens. La plupart des seigneurs, cependant, seraient satisfaits d'accepter un prix fixe pour la location simple de leurs fermes et de renoncer ainsi à perpétuité au paiement de toutes rentes, amendes et autres redevances féodales." Alors seulement on pourrait opérer le mélange des Anglais et des Canadiens. "Mais la loi telle qu'elle existe aujourd'hui, n'autorise pas cela; il faudrait un acte du parlement à l'effet de permettre le changement de tenure"s. Craig semble être resté sourd à la suggestion de Sewell. Aucune requête en ce sens ne fut présentée par lui au Parlement impérial.

George Prevost le remplaça en 1811 et son administration fut marquée par la guerre avec les États-Unis d'Amérique, suivie du traité de Gand en décembre 1814 et, quelques mois plus tard, de la fin des hostilités entre la France et la Grande-Bretagne et de la nomination de Gordon Drummond au poste de gouverneur.

7. Craig a Castlereagh, 15 juillet 1808, APC, Q 107: 197.

8. J. Sewell, "Remarques du juge en chef, de la réunion des provinces", [s.d. 1810 ], DRHC, $1791-1818,407$. 


\section{Caldwell veut commuer la tenure de ses terres}

Pendant que Napoléon apaisait ses instincts belliqueux sur l'île de son repos, la nation anglaise, faisant trêve à son activité fébrile des années de guerre, s'acheminait vers la dépression économique.

Quand en mil huit cent quinze, après la longue et sanglante contestation qui, pendant plus de vingt années, avoit agité et désolé l'Europe, il survint un nouvel ordre de choses, le Royaume Uni, de même que les autres nations de l'Europe, souffrit considérablement du passage subit d'un état de Guerre sans exemple dans l'histoire du genre-humain à un état de Paix. Son Gouvernement jetta naturellement les yeux sur les Colonies de l'Amérique Septentrionale comme sur un refuge pour ses Sujets en détresse et hors d'emploi. Les Propriétaires d'Angleterre espéroient que l'émigration des Pauvres dans les Colonies les soulageroit du poids énorme de la Loi faite en faveur des Pauvres. Les Pauvres eux-mêmes avoient droit de s'attendre à trouver, dans les vastes et immenses Terres incultes de la Couronne dans ces Colonies, un refuge et une honnête indépendance. Toutes les Classes pouvoient espérer que ce seroit un des moyens les plus puissants d'apaiser les mécontentements et de rétablir la tranquillitée.

"Dans la seule année 1815, on vit débarquer 700 émigrés écossais dans le port de Québec qui s'en allèrent jeter les bases de ce qui est aujourd'hui la ville florissante de Perth, dans la province d'Ontario. Et chaque année qui suivit apporta des contingents beaucoup plus considérables ${ }^{10}$."

John Caldwell, seigneur de Lauzon depuis la mort de son père Henry en $1810^{11}$, pensa tout de suite à retenir sur ses terres ces immigrants qui débarquaient tout près de chez lui ${ }^{12}$, mais qui s'em-

9. A. Stuart, "Quatrième rapport", 6 mars 1821, dans Premier rapport du Comite de la Chambre d'Assemblee sur cette partie de la harangue de Son Excellence le Gouverneur en chef, qui a rapport à l'etablissement des terres de la Couronne: avec les minutes des témoignages pris devant le Comité (Québec, 1821), 2: 37-38 [à l'avenir: Premier rapport ].

10. J.-E. Roy, Histoire de la seigneurie de Lauzon, 4: 38.

11. Ibid., 3: 430.

12. Ibid., 4: 39. 
pressaient de remonter le fleuve pour aller s'établir dans le HautCanada et aux Etats-Unis où ils retrouvaient leur propre langage et des lois et des institutions analogues à celles auxquelles ils avaient été accoutumés dans leur pays natal ${ }^{13}$.

Le premier obstacle à leur établissement dans le Bas-Canada résidait dans la tenure des terres et les lois françaises qui la régissaient. Ses affaires l'ayant poussé vers Londres à l'automne de $1815^{14}$, le seigneur de Lauzon en profita pour y discuter, avec les jurisconsultes de la couronne, la possibilité de changer la tenure de ses terres. On lui expliqua que le seul moyen possible d'effectuer la mutation désirée était de remettre ses terres entre les mains de Sa Majesté pour en recevoir une nouvelle concession en franc et commun soccage $^{15}$. Il s'adressa directement à Lord Bathurst ${ }^{16}$ et lui demanda la permission d'en user ainsi à l'égard des parties de ses seigneuries de Saint-Etienne et de Gaspé qui n'étaient pas encore concédées, soit les neuf-dixièmes des quarante mille arpents français que mesurait cette double seigneurie ${ }^{17}$.

Pourquoi y avait-il tant de terre inoccupée dans cette région, à une époque où les Canadiens se plaignaient de ne point trouver de terres dans les seigneuries? C'est que le fils Caldwell était avant tout un commerçant de bois et, lorsqu'il concédait une terre à un cultivateur, il commençait par s'approprier le bois marchand qui s'y trouvait. Il se réservait "le droit de couper ou faire couper, enlever ou faire enlever tous les bois de pin, épinette et autres propres à être sciés qui se pouvaient trouver tant sur les terres non concédées que sur les terres des différents tenanciers" 18 de ses seigneuries. Il spécifiait dans les contrats de concession qu'il gardait la propriété de "tous bois de pin, épinette, pruche et mérisier et autres propres à être sciés ou exploités"19, corrobore un contemporain. Résultat, les

13. A. Stuart, "Quatrième rapport", 6 mars 1821, dans Premier rapport, 2: 38.

14. J.-E. Roy, Histoire de la seigneurie de Lauzon, 4: 27.

15. "Requête de John Caldwell", 5 avril 1816, DRHC, 1791-1881, 496.

16. Bathurst à Drummond, 4 mai 1816, Ibid., 497.

17. "L'arpent de Paris équivaut à environ un sixième d'un acre anglais." Ibid., 497 note.

18. J.-E. Roy, Histoire de la seigneurie de Lauzon, 4: 105.

19. Jean-Paul, "Lettres seigneuriales à Agricola", La Minerve, 30 mars 1837. 
Canadiens lui laissèrent et son bois et ses terres et se dirigèrent vers celles de Gabriel-Elzéar Taschereau, seigneur de la NouvelleBeauce (seigneuries de Jolliet, Ste-Marie, St-Joseph) ${ }^{20}$.

Caldwell père, quoique plus modéré dans ses exigences, avait commencé à détourner les colons de sa seigneurie l'année où, faisant prendre titre nouvel à ses censitaires, il s'était réservé "le bois de chêne propre pour la construction des vaisseaux de Sa Majesté" ${ }^{21}$. Ce n'est pas par hasard que W. McKenzie pouvait écrire deux ans plus tard: "cette colonie... peut déjà fournir n'importent quelle grandeur et quelle quantité de mâts et de bois de chêne, aussi satisfaisants pour l'usage de la marine royale que celui qu'on peut se procurer n'importe où ailleurs" ${ }^{22}$. Durant les quatre années qui s'étendent de 1806 à 1810, écrit Joseph-Edmond Roy, les efforts du seigneur Caldwell semblent alors se diriger vers le commerce du bois plutôt que vers l'exploitation de ses terres ${ }^{23}$. Et Bouchette ajoute: "Depuis 1806 le commerce des colonies en bois de construction, mais surtout du Canada, s'est accru à un point extraordinaire;" de 1806 à 1810, les exportations se sont accrues de 100,000 à près de 375,000 tonneaux ${ }^{24}$. Voilà pourquoi, dans les seigneuries de Lauzon, de St-Etienne et de Gaspé, "la colonisation subit une espèce de ralentissement ${ }^{25}$ " durant les quatre années qui précédèrent la mort d'Henry Caldwell.

Rien d'étonnant si, après 1810 , les exigences du fils devenu seigneur éloignèrent les jeunes Canadiens. Aussi le "terrain cultivé" dans cette seigneurie en 1815 n'occupait-il qu'un "tiers de la totalité" ${ }^{26}$ de sa superficie. La même année, Bouchette notait: "Gaspé

20. J. Bouchette, Description Topographique de la province du Bas Canada, avec des remarques sur le Haut-Canada, et sur les relations des deux provinces avec Les EtatsUnis de l'Amérique, 517-518; J.-E. Roy, Histoire de la seigneurie de Lauzon, 3: 415.

21. Ibid., 3: 373-374.

22. "Mémoire de W. McKenzie", 12 février 1807, APC, Q 106-1: 56-57.

23. J.-E. Roy, Histoire de la seigneurie de Lauzon, 3: 415.

24. J. Bouchette, Description Topographique de la province du Bas Canada, avec des remarques sur le Haut Canada, et sur les relations des deux provinces avec Les Etats Unis de l'Amérique, 83-84.

25. J.-E. Roy, Histoire de la seigneurie de Lauzon, 3: 413. 502.

26. J. Bouchette, Description Topographique de la province du Bas Canada, 
ne renferme pas une seule acre en labour". Et, - ce qui confirme les témoignages ci-dessus - il ajoutait: "Tout ce terrain est abondamment pourvu de bois de construction, d'une bonne qualité, et l'on en coupe tous les ans une grande quantité qu'on envoie à Québec"'27. Dans ces conditions, le jeune seigneur jeta les yeux sur les immigrants britanniques pour peupler ses seigneuries.

Dans sa requête adressée à Lord Bathurst, il demandait donc qu'il fut donné au gouverneur général des Canadas, les instructions requises pour lui permettre d'opérer le changement qu'il avait en vue. La raison qu'il mettait de l'avant, était de nature à lui valoir une réponse affirmative: il était très désireux d'établir sur ses terres des émigrants britanniques; mais "ces terres étant tenues en vertu du système féodal... il s'ensuit irrémédiablement que toute personne du Royaume-Uni ne peut s'y établir dans de telles conditions"'28. Les hommes d'état anglais jugèrent l'idée excellente. Le secrétaire Bathurst s'empressa de donner des ordres: il s'agissait du bien-être de la communauté britannique. Monsieur, écrivait-il à Gordon Drummond, "vous voudrez bien prendre les mesures nécessaires pour recevoir de M. Caldwell, au nom de la couronne, et lui re-concéder ensuite, de la façon qu'il propose, les terres auxquelles il fait allusion dans sa requête ${ }^{29}$."

L'ordre était clair, la permission donnée sans restriction, le Gouverneur n'avait qu'à agir, l'intérêt britannique l'exigeait, quitte à préciser par la suite quelques points indécis. Mais la dépêche de Bathurst arriva dans un temps bien impropice. La dernière session avait été consacrée presque exclusivement à la discussion de l'affaire Sewell-Monk, le Parlement avait été dissous et le pays était en effervescence. Pour comble, le 12 juillet 1816, Gordon Drummond avait été remplacé par John Coape Sherbrooke ${ }^{30}$.

Le nouveau gouverneur procéda avec circonspection. Il demanda au Conseil exécutif d'étudier la question ${ }^{31}$. Heureusement pour les Canadiens, les conseillers trouvèrent toutes sortes d'objections à

27. Ibid., 510-511.

28. "Requête de John Caldwell", 5 avril 1816, DRHC, 1791-1818, 496.

29. Bathurst a Drummond, 4 mai 1816, Ibid., 497.

30. J. Neilson, éd., Journaux de la Chambre d'Assemblé du Bas-Canada, 24.

31. Cochran au juge en chef Sewell, 3 aout 1816, DRHC, 1791-1818, 497. 
la mutation de tenure demandée par Caldwell. Les droits de mutation ayant été donnés à la Province, on ne peut maintenant l'en priver sans la permission des représentants du peuple. Car en affranchissant ses terres de la tenure seigneuriale, Caldwell les affranchit à tout jamais de l'impôt du quint et prive ainsi la Province d'un revenu auquel elle a droit. Passe encore si le pétitionnaire offrait ou si le secrétaire d'état fixait une compensation. Le Conseil est donc d'avis qu'il appartient à l'Assemblée de fixer le montant de la compensation. Il croit de plus que ni l'enregistrement sur le papier terrier, ni une concession sous le grand sceau - comme cela se fait pour les terres non concédées de la couronne - ne suffirait à donner la validité à la mutation. Il soutient encore qu'il est absolument nécessaire de demander à la législature si la clause $36 \mathrm{du}$ statut britannique 31 Geo. III, chap. 31, exigeant la réserve d'un septième des terres concédées pour l'entretien du clergé protestant, doit s'appliquer à ces terres actuellement tenues en seigneurie. Et pour être bien sûr de ne rien oublier, le comité signalait que des ennuis très graves pourraient résulter de la commutation de la tenure d'une seigneurie hypothéquée. Le rapport se terminait par ces mots: "En somme, le comité croit qu'il est à propos de soumettre toute la question à la considération des ministres de Sa Majestét ${ }^{32}$."

En signant ce rapport à titre de président, Sewell y aurait bien ajouté une note de protestation à la façon d'Osgoode. Il s'en garda toutefois; mais sitôt sorti de la salle du Conseil, il entreprit de réfuter, dans une lettre personnelle au Gouverneur, tout ce qu'il avait dû signer à son corps défendant à titre de président du comité $\mathrm{du}$ Conseil. Il exposait ainsi son opinion: "je considère humblement qu'aucune loi ne s'y oppose". Puis il réfutait les objections du Conseil exécutif. Le roi de France pouvait recevoir une partie d'une seigneurie et la re-concéder en franc-alleu, pourquoi celui d'Angleterre n'aurait-il pas ce droit? Par ailleurs, que la couronne ait appliqué son revenu casuel et territorial à défrayer la dépense civile de la Province, cela ne lui enlève pas ses droits sur la propriété qui produit ce revenu. Et puis, que sont les 12,000 livres d'impôt que ces terres ont rapportées de 1791 à 1816, comparé aux avantages qui résulteraient d'un changement de tenure, lequel "aurait pour

32. "Rapport du comité du Conseil exécutif", 16 aout 1816, Ibid., 498-500. 
effet d'augmenter la population" en ouvrant les portes des seigneuries aux immigrants britanniques que repoussent actuellement les charges seigneuriales? Autres avantages: "les seigneurs deviendraient immédiatement de réels propriétaires et formeraient une aristocratie intéressée à la propriété foncière. Les fermiers anglais, écossais et irlandais seraient induits à cultiver les terres incultes des seigneurs actuels, dès qu'ils pourraient les obtenir libres de charges féodales. On verrait s'introduire un meilleur système d'agriculture"... Les cultivateurs, soulagés du droit de mutation et des autres charges féodales, seraient plus portés à faire des améliorations sur leur ferme. "Or, comme je suis d'avis à l'égard de ce qui précède que les instructions contenues dans la dépêche de lord Bathurst... sont clairement conformes à la loi et éminemment opportunes au point de vue politique, je ne puis admettre les raisons énoncées par le comité pour appuyer son rapport, bien que je considère en même temps qu'il est à propos de soumettre la mesure à la reconsidération de Sa Seigneurie conformément à l'opinion du comité" ${ }_{33}$.

Voilà le Gouverneur bien embarrassé. Qui a raison ? La majorité $\mathrm{du}$ Conseil contre son président, ou le président contre le Conseil ? Sherbrooke met les deux rapports contradictoires dans la même enveloppe $^{34}$ et les envoie outre-mer: les autorités prononceront entre les deux.

Après avoir étudié la question, les jurisconsultes rejettent l'opinion du Conseil exécutif. Les arguments de Sewell les ont convaincus: "Nous ne constatons, ni dans le statut 31, Geo. III, ch. 31, ni dans aucune loi en vigueur dans la province avant la conquête, aucun empêchement à ce que Sa Majesté accepte une remise de terres tenues en seigneuries et les concède de nouveau en franc et commun socage". "Néanmoins, nous considérons qu'il sera nécessaire lorsque se fera une nouvelle concession de ce genre d'accorder pour le maintien du clergé protestant, une partie proportionnelle d'une autre terre d'une valeur égale à la septième partie qui doit être déterminée dans la nouvelle concession ${ }^{35}$."

33. "Opinion du juge en chef Sewell au sujet du changement de tenure", 20 aout $1816, D R H C, 1791-1818,500-502$.

34. Sherbrooke à Bathurst, 24 aout 1816, APC, Q 137: 133.

35. W. Garrow et S. Shepherd, "Opinion des légistes au sujet du changement de tenure", 22 janvier $1817, D R H C, 1791-1818,504$. 
Il y a là un malentendu, pense le gouverneur à la lecture de ce rapport, et il en écrit à son supérieur: "Il ne s'agissait pas de savoir si la couronne a le pouvoir... mais si un tel changement de tenure en abolissant le droit de quint en ce qui concerne ces terres, droit transféré à la province par le message de lord Dorchester, ne constituerait pas en quelque sorte une violation de la parole donnée ainsi par le gouvernement; ou s'il était possible de donner à la province l'équivalent du droit de quint dont elle se trouverait dépouillée et privée par un tel changement de tenure ${ }^{36}$."

La lettre de Sherbrooke est remise aux légistes de la couronne. Ceux-ci l'étudient et y répondent. Dans leur premier rapport, disentils, ils se sont bornés à considérer le pouvoir de Sa Majesté d'opérer la mutation.

Mais la question actuellement soumise par la lettre du gouverneur est d'un caractère bien différent. Il ne s'agit plus du droit de la couronne de modifier la tenure mais de l'opportunité d'exercer de la sorte la prérogative de Sa Majesté, alors que la province sera ainsi dépouillée de l'une des sources de revenu appliquées au paiement des dépenses de son administration civile... Quant à ce point nous croyons que le message de lord Dorchester a donné droit à la province de s'attendre à ce que cette partie des revenus de Sa Majesté continue d'être appliquée au paiement des dépenses de l'administration civile de celle-ci et que lui enlever cette source de revenu sans son consentement ou sans lui accorder l'équivalent, serait une violation de ce qu'elle pourrait considérer comme une garantie ou une promesse de la part de la couronne.

Mais alors n'y aurait-il pas moyen de substituer une indemnité au paiement du droit de quint? "Toute source de revenu qui pourrait être créée à cet effet dans la province exigerait un acte législatif; et le consentement de la province à l'abolition du droit de quint ne pourrait être manifesté que par un acte de ce genre ou par quelque adresse des deux chambres à Sa Majesté. Nous pensions par conséquent que... un tel changement... sans le consentement de la législature provinciale manifesté comme susdit ou sans un équivalent, constituerait une violation de la promesse faite par le gouvernement dans le message. Et à ce point de vue Sa Majesté est empêchée, sans

36. Sherbrooke à Bathurst, 20 mai 1817, Ibid., 505. 
le consentement ou l'équivalent susmentionnés, de changer la tenure des terres ${ }^{37}$."

A la lettre contenant l'opinion des jurisconsultes, Bathurst ajouta ces mots: "Et pour les raisons énoncées dans celle-ci, [ inopportunité d'enlever à la Province sans son consentement cette source de revenu ] j'ai l'honneur de vous informer que, à mon avis, il ne serait pas à propos de changer la tenure des terres actuellement tenues en seigneurie ${ }^{38}$."

Si vraiment les Canadiens désiraient l'abolition de la tenure seigneuriale, il fallait porter la question devant la Chambre. Puisque, gagnée par la diplomatie de Sherbrooke, elle se montrait bien disposée, l'on pourrait faire des arrangements. Nouvelle preuve que telle n'était pas l'idée des Canadiens: on n'osa même pas en parler en public. L'attaque de 1815, dirigée par un seigneur anglais demandant une réforme favorable aux Britanniques, était détournée par le représentant même du roi, sans que les Canadiens aient eu à s'en mêler.

John Caldwell ne réussit pas à faire commuer la tenure de ses terres mais il parvint tout de même à garder dans Lauzon quelquesuns de ces immigrants ${ }^{39}$ qu'il aurait voulu attirer en foule sur ses domaines. Il en arrivait de pleins bateaux chaque été: "il est arrivé au Port de Québec, dans l'année 1817 six mille sept cent quatre-vingtseize Emigrés, en 1818 huit mille deux cent vingt et un, en 1819 douze mille neuf cent, sept en 1820 onze mille deux cent trente-neuf, formant en tout trente-neuf mille cent soixante et trois" ${ }^{40}$. De ce nombre, très peu s'établirent dans les seigneuries de Caldwell. L'étude du sort qu'il leur réserva nous fait voir, en miniature, ce que le seigneur de Lauzon rêvait d'accomplir en grand, s'il avait réussi à gagner son point. "La plupart furent employés par Caldwell comme manœuvres à l'exploitation de ses moulins ou à la coupe des bois ${ }^{41}$."

37. S. Shepherd et R. Gifford, "Deuxième rapport des jurisconsultes au sujet du changement de tenure", 1er aout 1817, DRHC, 1791-1818, 506.

38. Bathurst à Sherbrooke, 31 aout 1817, Ibid., 505.

39. J.-E. Roy, Histoire de la seigneurie de Lauzon, 4: 132.

40. A. Stuart, "Quatrième rapport", 6 mars 1821, dans Premier rapport, 2: 38.

41. J.-E. Roy, Histoire de la seigneurie de Lauzon, 4: 130. 
Ainsi, sous quelque côté qu'on envisage l'attaque de 1815 , on y verra un motif d'intérêt personnel de la part d'un seigneur, mais on ne pourra jamais dire: ce sont les censitaires qui ont demandé l'abolition de la tenure seigneuriale.

\section{Surpeuplement des environs de Québec}

A voir John Caldwell remuer ciel et terre pour attirer des immigrants dans ses seigneuries, on pourrait croire que la population de la région de Québec était trop peu considérable pour satisfaire aux besoins de la colonisation agricole. Si l'assertion semble contenir un peu de vrai appliquée aux seigneuries sises de part et d'autre de la Chaudière, elle devient tout à fait fausse lorsque nous traversons le Fleuve. Car, sur la rive nord, les Canadiens frappaient en vain à la porte des seigneurs pour en avoir des concessions ${ }^{42}$ : les bonnes terres à proximité de la ville étaient toutes occupées; “depuis 1800, jusqu'à la dernière guerre d'Europe, les progrès des établissemens dans les Seigneuries ont été considérables. Dans les endroits où les terres étoient bonnes, et pouvoient être obtenues à peu de frais,... elles étoient bientôt prises et établies, et les chemins tracés et faits à frais communs suivant la loi."

L'augmentation de la demande rendit les seigneurs indépendants et exigeants. "Les longs voyages, les délais, les frais et les difficultés pour obtenir des Procès-Verbaux, tant avant qu'après l'homologation, furent un sujet général de plainte: le haut prix des produits de l'agriculture, occasionné par la guerre en Europe, mit néanmoins les cultivateurs en état de tout supporter et de surmonter tous les obstacles qu'ils éprouvaient à faire des Chemins."

Il n'en fut plus ainsi après 1815 . En temps de guerre il n'est point d'obstacle qu'on ne surmonte; la paix revenue on perd cette lucidité et cette ténacité qui venaient à bout de tout pendant le conflit. Dès lors, la perspective d'avoir à tracer des chemins de plusieurs

42. "Par le mot concessions on entend des lots de terre ordinairement de trois arpents de front sur 20,30, ou 40 de profondeur que les seigneurs louent pour une rente modique, en argent ou en nature, suivant leur qualité, à des personnes qui veulent bien s'y établir et les cultiver." J. Bouchette, Description Topographique de la province du Bas Canada, avec des remarques sur le Haut Canada, et sur les relations des deux provinces avec Les Etats Unis de l'Amérique, 93 note. 
milles de longueur, apparut comme un obstacle insurmontable. "Depuis la fin de la guerre les progrès des établissements ont diminué d'année en année."

Par bonheur, le député John Neilson était un homme d'action. Il fit plus que des promesses, mieux qu'un beau discours, il fit une œuvre. Bel exemple de dévouement, il prit lui-même l'initiative de trouver des terres pour les jeunes et assuma lui-même la tâche d'établir les nouveaux colons. A cette fin, il s'assura la coopération de ses amis Andrew Stuart et Louis Moquin, tous deux avocats de Québec comme lui, et ensemble, ils étudièrent le problème.

L'étendue de terre sur laquelle ils jetèrent les yeux, faisait partie du fief Saint-Gabriel, propriété du gouvernement depuis la mort du dernier Jésuite en 1801 et dans lequel aucune concession n'avait été faite depuis ce temps. Elle était située à une distance de six à neuf milles de toute habitation. Aucun chemin ne s'y rendait; un sentier en forêt en était la seule voie d'accès, encore traversait-il un marais où le passant avait de l'eau aux genoux en toute saison.

Qu'à cela ne tienne, on s'y rendit. L'endroit fut jugé propice à la culture. Les trois avocats passèrent ensuite chez les Commissaires des biens des Jésuites et en obtinrent cinquante concessions adjacentes de trois arpents par trente, en roture, aux rentes et conditions ordinaires ${ }^{43}$. Les cens et rentes étaient donc ceux en usage sous le régime français. Conditions ordinaires signifiait "que quatre arpens et demi soient mis en culture et qu'il y ait une maison de bâtie dans l'année". C'était un beau succès pour ces hommes; aussi pouvaient-ils dire des Commissaires: "Leurs Concessions ne sont pas onéreuses ni alarmantes pour ceux qui s'[ y ] établissent ${ }^{44}$."

Travaux d'arpentage, obtention des titres, tracé des chemins, érection des ponts, construction du moulin à scie, défrichage des quelques lots restants, tout se fit "aux frais et sous la surveillance personnelle" de ces trois hommes. Ils accomplirent cette tâche

43. Témoignage de John Neilson, 31 décembre 1823, Appendice du $X X X I I I e$ volume des Journaux de la Chambre d'Assemblée de la province du Bas-Canada [Québec, 1824 ], Appendice R, Appendice A [ n. p. ]. Les citations précédentes sont tirées de ce document.

44. "Plan par John Neilson, Ecuyer, pour former un nouvel Établissement étendu sur le côté nord du Fleuve Saint-Laurent, dans les environs de Québec", octobre 1819, Appendice du XXXIIIe volume des Journaux de la Chambre d'Assemblee de la province du Bas-Canada, Appendice R, Appendice A. 
onéreuse sans espoir de rémunération ${ }^{45}$. Leurs efforts réunis pendant trois années, assurèrent le succès de cet établissement, connu sous le nom de Val-Cartier, sans que cela ait coûté un seul denier au propriétaire du fief ${ }^{46}$.

Non seulement la Commission des biens des Jésuites ne fit rien pour contribuer au succès de cette entreprise, elle en arrêta l'essor. Une fois le gros œuvre accompli et les colons bien installés, d'autres Canadiens demandèrent à prendre des lots adjacents aux cinquante premiers: la Commission refusa ${ }^{47}$. Pourquoi? Il nous suffit de voir le nom de $\mathrm{H}$. W. Ryland à la tête de la Commission des biens des Jésuites - dont dépendait le fief St-Gabriel - pour nous expliquer cet entêtement. Ayant eu soin de s'entourer exclusivement d'anglophones ${ }^{48}$, le président pouvait facilement leur inoculer ses sentiments. Or, le fait est notoire, Ryland n'aimait pas les Canadiens. Cette Commission se laissa fléchir, il est vrai, en 1820 mais pour cause. La demande venait d'Andrew William Cochran, président de la Société de Secours aux Emigrés et avait pour but de secourir des Irlandais et des Écossais ${ }^{49}$. C'est ainsi que des étrangers occupèrent les terres encore disponibles dans cette région ouverte aux frais de John Neilson et de ses amis et dont la seule voie d'accès était la route faite par leurs soins.

Il semble, d'après les paroles de John Neilson, que d'autres particuliers prirent aussi l'initiative d'ouvrir des chemins vers des terres neuves. Il dit en effet: "Les autres établissements dans Stoneham, et Beauport, ont été effectués principalement avec le capital de citoyens de Québec"50. Qu'est-ce à dire sinon que les seigneurs ne s'acquittaient plus de leurs fonctions et laissaient les colons se

45. Témoignage de John Neilson, 31 décembre 1823, Ibid.

46. "Plan par John Neilson, Ecuyer, pour former un nouvel Ettablissement étendu sur le côté nord du Fleuve Saint-Laurent, dans les environs de Québec"“ octobre 1819, Ibid.,

47. Témoignage de John Neilson, 31 décembre 1823, Ibid.

48. A. Stuart, "Quatrième rapport", 6 mars 1821, dans Premier rapport, 2: 41.

49. Ibid., 41-42.

50. Témoignage de John Neilson, 31 décembre 1823, Appendice du XXXIIIe volume des Journaux de la Chambre d'Assemblée de la province du Bas-Canada, Appendice R, Appendice A. 
débrouiller comme ils le pouvaient? Mais ces quelques établissements ne pouvaient suffire aux besoins de la région de Québec où les Canadiens étaient à l'œuvre depuis déjà deux cents ans et dont la population avait considérablement augmenté depuis le temps de Louis Hébert. Les terres en bordure du St-Laurent une fois occupées, les nouvelles générations avaient poussé le défrichement toujours plus loin du fleuve, traçant de nouveaux rangs à l'arrière des premières côtes. Et l'on se trouvait maintenant, en plusieurs endroits, acculé aux Laurentides. "Les Montagnes offrent des barrières insurmontables pour le présent à l'extension des Etablissements qu'elles bordent;" ailleurs, des marécages rendaient la prolongation des chemins très difficile; "mais, écrivait Neilson en évoquant son succès à Val-Cartier, les Terres basses et marécageuses sont des obstacles qui peuvent aisément être et qui en effet ont déjà été surmontés ${ }^{51}$."

On le voit, la terre facile d'accès était occupée. Pour en avoir, désormais, il faudrait tracer des chemins longs et coûteux. John Neilson prit encore les devants. Il rédigea un plan en vue d'ouvrir une nouvelle région à l'agriculture. "Il y a moyen, y lit-on, de trouver de la bonne terre derrière les montagnes et les marais, en traçant des chemins le long des Rivières". Jusqu'à ce jour, "pour les causes ci-dessus mentionnées", les établissements se sont rarement étendus à plus de deux ou trois lieues du Fleuve. En allant en amont de la rivière Jacques-Cartier jusqu'à cinq ou six lieues du fleuve StLaurent, on trouvera "une étendue de Terre montagneuse" où l'on pourra établir les gens sans terre. Le grand obstacle est l'absence de chemin, mais "en faisant ouvrir quatre lieues de chemin de voiture" pour se rendre à Val-Cartier, "en cultivant sur les lieux assez pour supporter les travaillans; en un mot, en mettant des travaillans et des provisions quatre lieues avant dans les forêts, dans la direction de l'étendue de Terre qu'on propose d'ouvrir maintenant, la partie la plus difficile de l'ouvrage a été faite."

Qui s'occupera de faire tracer l'autre bout de chemin ? Faudra-til encore compter sur l'initiat ve privée? Neilson n'est tout de même pas le grand voyer et les avocats de Québec n'ont pas les

51. "Plan par John Neilson, Ecuyer, pour former un nouvel Etablissement étentu sur le côté nord du Fleuve Saint-Laurent, dans les environs de Québec", octobre 1819, Ibid., 
moyens de financer tous les travaux de voirie de la région. La Province? "D'après la mauvaise réussite de l'expérience qui a été faite récemment d'ouvrir de nouveaux Chemins avec l'Argent public, il n'est pas probable, ni peut-être à souhaiter, qu'on obtienne aucune assistance de la Législature pour cet objet." Remarquons ici l'hésitation de l'avocat député: "il n'est pas probable, n' peut-être à souhaiter...52" Tout de même, il ne peut s'empêcher d'en parler: preuve qu'il a hésité à se prononcer dans une dure alternative, et qu'il serait encore disposé à se laisser convaincre du contraire. Et de fait, est-ce que l'argent du peuple n'aurait pas été plus utile au peuple s'il avait servi à ouvrir des routes, qu'employé à améliorer les propriétés du receveur général ${ }^{53}$ ?

Puisqu'il ne fallait pas compter sur un subside du gouvernement, John Neilson pensa à demander la collaboration des seigneurs de la région: "Les individus dont la valeur des propriétés seroit augmentee par l'ouverture de ces Chemins devraient pourvoir aux moyens de les faire et $\mathrm{y}$ prendre une part active, ainsi qu'ils y sont tenus par les conditions des anciennes Concessions en Seigneuries, et il est rare qu'aucune autre manière réussisse" ${ }^{54}$. Un rapport, rédigé à la Chambre quelques années plus tard, notait aussi que: "Le Seigneur dans certains cas, mais non universellement, étoit tenu, par les conditions de la Concession originaire, d'ouvrir un Chemin pour communiquer des Établissements de sa Seigneurie les plus éloignés a la Concession suivante des terres non-concédées dans icelle"55. La précaution de l'avocat Neilson à spécifier "anciennes Concessions" semble indiquer que c'est à cette catégorie que s'applique la clause ci-dessus. Il estimait pour sa part, que la collaboration à l'ouverture

52. Ibid.

53. A cette époque, l'argent perçu par le receveur général était confié à sa garde. Il en avait même la jouissance tant que la Province n'en avait pas besoin. John Caldwell en profita pour construire des moulins à scie et mener grand train. Un jour vint où il dut s'avouer insolvable. Le soi-disant trésor amassé, préservé et défendu avec un soin jaloux par nos bons députés, était dilapidé. - Sur ce sujet, voir: J.-E. Roy, Histoire de la seigneurie de Lauzon, 4: 279-406.

54. "Plan par John Neilson, Écuyer, pour former un nouvel Établissement étendu sur le côté nord du Fleuve Saint-Laurent, dans les environs de Québec", octobre 1819, Appendice du XXXIIIe volume des Journaux de la Chambre d'Assemblée de la province du Bas-Canada, Appendice R, Appendice A.

55. A. Stuart, "Premier rapport", 12 février 1821, dans Premier rapport, 1: 9. 
du chemin demandé, s'imposait comme un devoir aux seigneurs de cette région. Les intéressés étaient: Juchereau Duchesnay, seigneur de Faussembault, B. Panet, seigneur de Bourg-Louis, d'Auteuil, seigneur du fief de ce nom, Allsop, seigneur de Jacques-Cartier, C.-E. C. de Léry, seigneur de Perthuis ${ }^{56}$.

Le point délicat serait d'obtenir leur adhésion. Ce n'était pas chose facile. "Un des plus grands obstacles à l'extension des Chemins et des Établissements nouveaux... est la conduite indifférente ou mesquine de quelques propriétaires de grandes étendues de Terres incultes. Ils ne veulent se donner aucune peine, ne donnent aucune facilité, mais dès que leurs Terres viennent à avoir du prix, par le moyen des Chemins ou des nouveaux Établissemens faits aux frais ou par les efforts d'autres personnes ils exigent pour leurs Concessions des conditions plus onéreuses, ce qui, avec les difficultés et la perte de tems que l'on éprouve souvent avant d'obtenir les Concessions, décourage les personnes qui veulent s'établir ${ }^{57}$."

Si ces obstacles ne découragèrent pas l'auteur même de ce plan, du moins l'empêchèrent-ils de le mettre à exécution. Rédigé en 1819, il était encore à l'état de projet lors de l'enquête du Comité des Terres de la Couronne en $1823^{58}$.

Le jeu en aurait-il valu la chandelle? C'est une autre question. Il reste acquis qu'un malaise se fait sentir à l'intérieur de la zone seigneuriale. La fondation de Val-Cartier en 1816 et le plan de 1819 ont une valeur symptomatique: la colonisation agricole est à la gêne au moins dans le voisinage de Québec.

Frère MARCEL-Joseph, S.G., M.A.

(à suivre)

56. J. Bouchette, Description Topographique de la province du Bas Canada, avec des remarques sur le Haut Canada, et sur les relations des deux provinces avec Les Etats Unis de l'Amérique, 414; "Plan par John Neilson, Écuyer, pour former un nouvel Etablissement étendu sur le côté nord du Fleuve Saint-Laurent, dans les environs de Québec", octobre 1819, Appendice du XXXII Ie volume des Journaux de la Chambre d'Assemblée de la province du Bas-Canada, Appendice R, Appendice A.

57. Ibid.

58. Témoignage de John Neilson, 31 décembre 1823, Ibid. 